\title{
HODGE COHOMOLOGY OF SOME FOLIATED BOUNDARY AND FOLIATED CUSP METRICS
}

\author{
JESSE GELL-REDMAN AND FRÉDÉRIC ROCHON
}

\begin{abstract}
For fibred boundary and fibred cusp metrics, Hausel, Hunsicker, and Mazzeo identified the space of $L^{2}$ harmonic forms of fixed degree with the images of maps between intersection cohomology groups of an associated stratified space obtained by collapsing the fibres of the fibration at infinity onto its base. In the present paper, we obtain a generalization of this result to situations where, rather than a fibration at infinity, there is a Riemannian foliation with compact leaves admitting a resolution by a fibration. If the associated stratified space (obtained now by collapsing the leaves of the foliation) is a Witt space and if the metric considered is a foliated cusp metric, then no such resolution is required.
\end{abstract}

\section{Contents}

1. Introduction

2. Seifert fibrations and stratified spaces

3. From intersection cohomology to weighted cohomology

4. From weighted cohomology to $L^{2}$ cohomology.

5. Proofs of main theorems

6. Some examples

References

\section{INTRODUCTION}

The Hodge theorem states that for a complete, compact Riemannian manifold without boundary, the space of $L^{2}$ harmonic forms - the 'Hodge cohomology' - is isomorphic to the de Rham cohomology. For manifolds that are either not complete or not compact, no general relationship between the Hodge cohomology and a topological invariant is known, but there is a wealth of Hodge type theorems in various settings: on manifolds with cylindrical ends [2], on singular algebraic varieties [6], on locally symmetric spaces [31], [26], on asymptotically geometrically finite hyperbolic quotients [18], [19], and the well-known work of Cheeger [5] and Nagase [21] (see also [4]), which relates the Hodge cohomology of manifolds with iterated conical singularities with the intersection cohomology groups of Goresky and Macpherson, [12], [13]. These same intersection cohomology groups appear in the work of Hausel, Hunsicker, and Mazzeo [14] on the Hodge cohomology of fibred boundary and fibred cusp metrics, two natural geometries defined on a smooth manifold whose boundary is diffeomorphic to a fibration.

Our goal in this paper is to extend the results of Hausel, Hunsicker, and Mazzeo to the more general case where the boundary is diffeomorphic to a Seifert fibration. A Seifert fibration is, loosely speaking, a foliation whose space of leaves is an orbifold. See Section 2 for a detailed description. On such manifolds, fibred boundary and fibred cusp metrics have 
natural analogues: foliated boundary and foliated cusp metrics as introduced in [24], and it is these metrics whose Hodge cohomology we study here. To keep the analysis part of the paper tractable, we will further assume that the Seifert fibration is good, meaning that the space of leaves of the boundary foliation is the quotient of a smooth compact manifold by a smooth, properly discontinuous action of a finite group. This condition is needed only in Section 4, and we hope to remove it in a future work.

Thus, let $M$ be a non-compact manifold inside a compact, smooth manifold with boundary $\bar{M}$, whose boundary $\partial M=\bar{M}-M$ is the total space of a Seifert fibration $\mathcal{F}$, for the moment not necessarily good. Let $B$ denote the space of leaves of $\mathcal{F}$, and let

$$
\pi: \partial M \longrightarrow B
$$

be the associated projection. Let $x$ be a boundary defining function (b.d.f.) for $\partial M$, i.e. $x \in C^{\infty}(\bar{M})$ with $x^{-1}(0)=\partial M,\left.d x\right|_{\partial M} \neq 0$ and $x>0$ on $M$. For small $\epsilon>0$, the set $x^{-1}([0, \epsilon))$ is diffeomorphic to $\partial M \times[0, \epsilon)_{x}$, and we extend the projection $\pi$ to this neighborhood of the boundary in the obvious way. Then an exact foliated boundary metric is a Riemannian metric which on $\partial M \times[0, \epsilon)_{x}$ takes the form

$$
g_{\mathcal{F}}=\frac{d x^{2}}{x^{4}}+\frac{\pi^{*} h}{x^{2}}+k
$$

where $h$ is an orbifold Riemannian metric on $B$ and $k$ is a $(0,2)$-tensor which restricts to a Riemannian metric on each leaf of the foliation $\mathcal{F}$. Similarly, an exact foliated cusp metric is a metric of the form

$$
g_{\mathcal{F}-c}:=\frac{d x^{2}}{x^{2}}+\pi^{*} h+x^{2} k
$$

with $h$ and $k$ as above. General (i.e. non-exact) foliated metrics are permitted to have "crossterms"; see Section 3. When the base $B$ of the Seifert fibration is smooth, $\mathcal{F}$ corresponds to the fibres of a smooth fibration, and we recover from these definitions the notion of exact fibred boundary metric and exact fibred cusp metric considered for instance in [14].

For a choice of foliated boundary or foliated cusp metric $g$ on $M$, let $L^{2} \mathcal{H}^{k}(M, g)$ denote the space of $L^{2}$ harmonic forms of degree $k$, i.e. those $\omega \in \Omega^{k}(M)$ with $\|\omega\|_{L^{2}(g)}<\infty$ solving $d \omega=0$ and $\delta \omega=0$, where $\delta$ is the adjoint of $d$ with respect to the $L^{2}$ norm induced by $g$. To relate $L^{2} \mathcal{H}^{k}(M, g)$ with some topological data, we consider, instead of $M$, the space $X$ obtained by collapsing the leaves of the foliation on $\partial M$ onto the space of leaves $B$. To be precise, let

$$
X:=\bar{M} / \sim \text {, where } p \sim q \Longleftrightarrow p=q \text { or } p, q \in \partial M \text { with } \pi(p)=\pi(q) .
$$

There is a corresponding collapsing map $c_{\pi}: \bar{M} \rightarrow X$ which is the identity on $M$ and is given by the projection $\pi$ on $\partial M$. We identify $B$ with $c_{\pi}(\partial M) \subset X$. The space $X$ is a (smoothly) stratified space (see for instance [1] or [8] for a definition), and as such, the intersection homology and cohomology groups of Goresky and MacPherson [12] can be defined thereon. These groups are not homotopically invariant like singular homology and cohomology groups, but they are topological invariants. They are defined in terms of a perversity function, i.e. a map $\mathfrak{p}:\{0,1, \ldots, n\} \longrightarrow \mathbb{N}$ satisfying $\mathfrak{p}(\ell) \leq \mathfrak{p}(\ell+1) \leq \mathfrak{p}(\ell)+1$, and a stratification, i.e. is a nested sequence

$$
X \supset X_{n-2} \supset \cdots \supset X_{j} \supset \cdots \supset X_{0},
$$


where $X-X_{n-2}$ is a smooth manifold and $X_{j}-X_{j-1}$ is either empty or a manifold of dimension $j$. The group $I H_{\mathfrak{p}}^{k}(X)$ is the $k^{\text {th }}$ cohomology group of the complex of cochains defined on chains which intersect each stratum of codimension $\ell$ in a set of dimension at most $k-\ell+\mathfrak{p}(\ell)$. The original intersection homology theory was developed for standard perversities, i.e. those satisfying the additional condition $\mathfrak{p}(0)=\mathfrak{p}(1)=0$, but general perversities are now in common use, see e.g. [14], [22]. For standard perversities, the group $I H_{\mathfrak{p}}^{k}(X)$ turns out to be independent of the stratification. For more on intersection cohomology, see [3].

Let

$$
\begin{aligned}
n & :=\operatorname{dim} M \\
b & :=\operatorname{dim} B \\
f & :=n-b-1,
\end{aligned}
$$

so $f$ is the dimension of a typical leaf of $\mathcal{F}$. As we discuss in Section 2, $X$ carries a natural stratification induced by the orbifold structure of the space of leaves, $B$. In particular, the spaces $X_{n-2}, \ldots, X_{n-(f+1)}$ are all equal to $B$. In Corollary 17 below, we show that the intersection cohomology of $X$ depends only on $\mathfrak{p}(f+1)$. Thus, the following definition makes sense. For $j \in \mathbb{N}$, let

$$
I H_{j}^{k}(X, B):=\left\{\begin{array}{ccc}
H^{k}(X-B) & \text { if } & j \leq-1 \\
I H_{\mathfrak{p}}^{k}(X) \text { where } \mathfrak{p}(f+1)=j . & \text { if } & 0 \leq j \leq f-1 \\
H^{k}(X, B) & \text { if } & f \leq j
\end{array}\right.
$$

c.f. Section 2.2, equation (9) of [14].

Theorem 1. Let $\bar{M}$ be a manifold with boundary, $\partial M=\bar{M}-M$. Let $\mathcal{F}$ be a good Seifert fibration on $\partial M$, and let $g_{\mathcal{F}}$ be a foliated boundary metric on $M$. Then for any degree $0 \leq k \leq n$, there are natural isomorphisms

$$
L^{2} \mathcal{H}^{k}\left(M, g_{\mathcal{F}}\right) \longrightarrow \begin{cases}\operatorname{Im}\left(I H_{f+\frac{b+1}{2}-k}^{k}(X, B) \longrightarrow I H_{f+\frac{b-1}{2}-k}^{k}(X, B)\right) & b \text { odd } \\ I H_{f+\frac{b}{2}-k}^{k}(X, B) & b \text { even },\end{cases}
$$

with $B$ as in (1) and $X$ as in (4).

Theorem 2. Notation and assumptions as in Theorem 1, let $g_{\mathcal{F}-c}$ be a foliated cusp metric on $M$. Then for $0 \leq k \leq n$, there is a natural isomorphism

$$
L^{2} \mathcal{H}^{k}\left(M, g_{\mathcal{F}-c}\right) \longrightarrow \operatorname{Im}\left(I H_{\mathfrak{m}}^{k}(X, B) \longrightarrow I H_{\mathfrak{m}}^{k}(X, B)\right)
$$

where $\underline{\mathfrak{m}}$ and $\overline{\mathfrak{m}}$ are the lower middle and upper middle perversities, (see Section 5 .)

As in [14], when $X$ is a Witt space (again, see Section 5), the result simplifies, since $I H_{\mathfrak{m}}^{k}(X, B)=I H_{\mathfrak{m}}^{k}(X, B)$, and the proof involves softer analytical methods. In particular, it does not requires the Seifert fibration $\mathcal{F}$ to be good. This gives the following.

Theorem 3. Let $\bar{M}$ be a manifold with boundary, $\partial M=\bar{M}-M$. Let $\mathcal{F}$ be a Seifert fibration on $\partial M$, and let $g_{\mathcal{F}-c}$ be a foliated cusp metric on $M$. If the space $X$ described above is Witt, then there are natural isomorphisms

$$
L^{2} \mathcal{H}^{*}\left(M, g_{\mathcal{F}-c}\right) \cong H_{(2)}^{*}\left(M, g_{\mathcal{F}-c}\right) \cong I H_{\underline{\mathfrak{m}}}^{*}(X, B),
$$

where $H_{(2)}^{*}\left(M, g_{\mathcal{F}-c}\right)$ is the $L^{2}$-cohomology of $\left(M, g_{\mathcal{F}-c}\right)$. 
When the boundary foliation is induced by a fibration, these theorems reduce to the results of [14]. Moreover, Theorems 1 and 2 simplify if the typical leaf is a quotient of a sphere by the free, properly discontinuous action of a finite group, for then $X$ itself is an orbifold. Since the intersection cohomology of an orbifold is isomorphic to the singular cohomology for any standard perversity (a well-known fact, see Theorem 19 below), this leads to the following analogues of Corollaries 1 and 2 from [14].

Corollary 4. Let $\left(M, g_{\mathcal{F}}\right)$ satisfy the assumptions of Theorem 1 and assume that the typical leaf is the quotient of a sphere by the free, properly discontinuous action of a finite group. Then for any degree $0 \leq k \leq n$, there are natural isomorphisms

$$
L^{2} \mathcal{H}^{k}\left(M, g_{\mathcal{F}}\right) \cong \begin{cases}H^{k}(X, B) & k \leq \frac{b-1}{2} \\ \operatorname{Im}\left(H^{k}(X, B) \longrightarrow H^{k}(X)\right) & k=\frac{b-1}{2}+1 \\ H^{k}(X) & \frac{b+1}{2}<k<n-\frac{b+1}{2} \\ \operatorname{Im}\left(H^{k}(X) \longrightarrow H^{k}(X \backslash B)\right) & k=n-\frac{b+1}{2} \\ H^{k}(X \backslash B) & k \geq n-\frac{b-1}{2}\end{cases}
$$

if $b$ is odd, and

$$
L^{2} \mathcal{H}^{k}\left(M, g_{\mathcal{F}}\right) \cong \begin{cases}H^{k}(X, B) & k \leq \frac{b}{2} \\ H^{k}(X) & \frac{b}{2}<k<n-\frac{b}{2} \\ H^{k}(X \backslash B) & k \geq n-\frac{b}{2}\end{cases}
$$

if $b$ is even.

Corollary 5. Let $\left(M, g_{\mathcal{F}-c}\right)$ satisfy the assumptions of Theorem 3 and assume that the typical leaf is the quotient of a sphere by the free properly discontinuous action of a finite group. Then for any degree $0 \leq k \leq n$,

$$
L^{2} \mathcal{H}^{k}\left(M, g_{\mathcal{F}-c}\right) \cong H_{(2)}^{*}\left(M, g_{\mathcal{F}-c}\right) \cong H^{k}(X) .
$$

The structure of the present paper is closely related to that of [14]. There, the authors begin by identifying the intersection cohomology groups with the cohomology of a complex of weighted differential forms. They then equate the Hodge cohomology with the images of maps of certain weighted cohomology groups. Combining these two results gives Theorems 1 and 2 in the fibration case. The lion's share of the work, and all of the analysis, is in the second step. Here, the reverse is true; most of the work lies in identifying intersection cohomology with weighted cohomology, whereas the identification of Hodge cohomology with (images of maps of) weighted cohomology follows directly from the arguments in [14], which we use essentially as a black box.

Acknowledgements. We are grateful to Markus Banagl and Eugénie Hunsicker for some helpful correspondence.

\section{SEIfERT Fibrations AND Stratified spaCES}

As in [11], by "Seifert fibration", we mean the natural generalization of Seifert fibrations on 3-manifolds to arbitrary dimensions. A Seifert fibration on a compact manifold $M$ is, simply put, a Riemannian foliation $\mathcal{F}$ with compact leaves. Since the space of leaves has naturally the structure of an effective orbifold, see for instance [11] or [20, there is an alternative definition involving orbibundles, which we describe now following [11]. First, we recall the definition of an orbifold (see for instance [27]). 
Definition 6. An n-dimensional smooth orbifold is a second countable Hausdorff space B such that:

(i) $B$ has a covering by a collection of open sets $\left\{\mathcal{U}_{i}\right\}$ closed under finite intersections. For each $\mathcal{U}_{i}$, there is a finite group $\Gamma_{i}$ with a smooth action (on the right) on an open subset $\widetilde{\mathcal{U}}_{i} \subset \mathbb{R}^{n}$ and a homeomorphism $\varphi_{i}: \widetilde{\mathcal{U}}_{i} / \Gamma_{i} \rightarrow \mathcal{U}_{i}$.

(ii) Whenever $\mathcal{U}_{i} \subset \mathcal{U}_{j}$, there is an injective homomorphism $\nu_{i j}: \Gamma_{i} \rightarrow \Gamma_{j}$ and an embed$\operatorname{ding} \widetilde{\varphi}_{i j}: \widetilde{\mathcal{U}}_{i} \rightarrow \widetilde{\mathcal{U}}_{j}$ equivariant with respect to $\nu_{i j}$ and making the following diagram commute,



The quadruple $\left(\mathcal{U}_{i}, \widetilde{\mathcal{U}}_{i}, \Gamma_{i}, \varphi_{i}\right)$ is called an orbifold chart and the collection of orbifold charts is called an orbifold atlas.

We say an orbifold is effective if, for all orbifold charts $\left(\mathcal{U}_{i}, \widetilde{\mathcal{U}}_{i}, \Gamma_{i}, \varphi_{i}\right)$, the action of $\Gamma_{i}$ on $\widetilde{\mathcal{U}}_{i}$ is effective, i.e. the identity of $\Gamma_{i}$ is the only element which acts as the identity map. On an orbifold, we can consider bundles which are compatible with the orbifold structure.

Definition 7. Let $B$ be an orbifold and $\widetilde{F}$ a smooth manifold. Then a smooth orbibundle with fibre $\widetilde{F}$ is an orbifold $Y$ together with a surjective continuous map $\pi: Y \rightarrow B$ satisfying the following:

(i) $B$ admits an orbifold atlas of orbifold charts $\left(\mathcal{U}_{i}, \widetilde{\mathcal{U}}_{i}, \Gamma_{i}, \varphi_{i}\right)$ such that for each $i$, there is a smooth action of $\Gamma_{i}$ on $\widetilde{\mathcal{U}}_{i} \times \widetilde{F}$ making the projection $\widetilde{\mathcal{U}}_{i} \times \widetilde{F} \rightarrow \widetilde{\mathcal{U}}_{i} \Gamma_{i}$-equivariant, and there is a homeomorphism $\psi_{i}:\left(\widetilde{\mathcal{U}}_{i} \times \widetilde{F}\right) / \Gamma_{i} \rightarrow \pi^{-1}\left(\mathcal{U}_{i}\right)$ inducing the following commutative diagram,

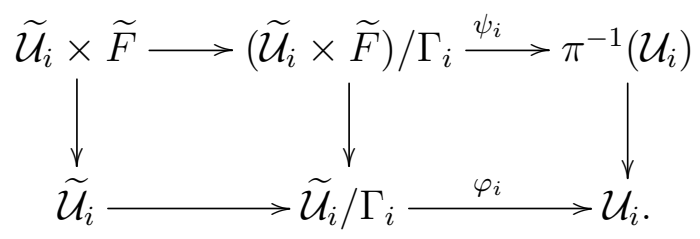

(ii) Whenever $\mathcal{U}_{i} \subset \mathcal{U}_{j}$, there exists a $\Gamma_{i}$-equivariant embedding $\widetilde{\psi}_{i j}: \widetilde{\mathcal{U}}_{i} \times \widetilde{F} \rightarrow \widetilde{\mathcal{U}}_{j} \times \widetilde{F}$ making the following diagram commutes,

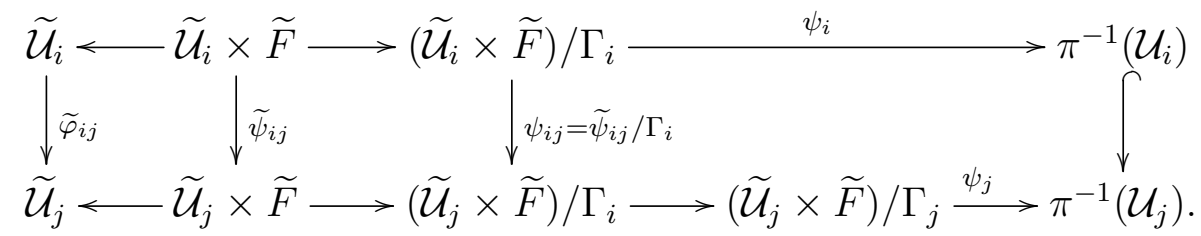

In particular, the collection $\left(\pi^{-1}\left(\mathcal{U}_{i}\right), \widetilde{\mathcal{U}}_{i} \times \widetilde{F}, \Gamma_{i}, \psi_{i}\right)$ form an orbifold atlas for $Y$.

Remark 8. Without loss of generality, we can assume that in the orbifold chart $\left(\pi^{-1}\left(\mathcal{U}_{i}\right), \widetilde{\mathcal{U}}_{i} \times\right.$ $\left.\widetilde{F}, \Gamma_{i}, \psi_{i}\right)$, the action of $\Gamma_{i}$ on $\widetilde{\mathcal{U}}_{i} \times \widetilde{F}$ is induced by the action on $\widetilde{\mathcal{U}}_{i}$ and a fixed action on $\widetilde{F}$,

$$
(u, f) \cdot \gamma=(u \cdot \gamma, f \cdot \gamma), \quad u \in \widetilde{\mathcal{U}}_{i}, f \in \widetilde{F}, \gamma \in \Gamma_{i} .
$$


Indeed, if the action is not already of this form, one can use a $\Gamma_{i}$-equivariant connection for the fibration $\widetilde{\mathcal{U}}_{i} \times \widetilde{F} \rightarrow \widetilde{\mathcal{U}}_{i}$ and parallel transport to identify each fibre above $\widetilde{\mathcal{U}}_{i}$ with a fixed one. With such an identification, the action of $\Gamma_{i}$ is of the desired form.

We can now define Seifert fibrations in terms of orbibundles.

Definition 9. A Seifert fibration is an orbibundle for which the total space $Y$ is a smooth manifold, that is, such that the $\Gamma_{i}$ actions on $\widetilde{\mathcal{U}}_{i} \times \widetilde{F}$ are free for each $i$.

Remark 10. As explained in [11, Remark 1.3], there is no loss of generality in assuming that the space of leaves of a Seifert fibration is an effective orbifold. For this reason, we will assume that all the orbifolds considered are effective unless otherwise specified.

In Section 4, we will consider the following special case.

Definition 11. A foliation $\mathcal{F}$ on $Y$ is a good Seifert fibration if it arises as follows:

(i) $Y=\widetilde{Y} / \Gamma$ where $\widetilde{Y}$ is a smooth compact manifold on which a finite group $\Gamma$ acts on the right by diffeomorphisms freely and properly discontinuously. The manifold $\widetilde{Y}$ is the total space of a fibration

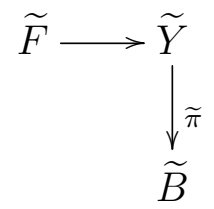

where the base $\widetilde{B}$ is a closed manifold and the fibre $\widetilde{F}$ is a smooth manifold. The group $\Gamma$ acts smoothly on $\widetilde{B}$ in such a way that

$$
\widetilde{\pi}(y \cdot \gamma)=\tilde{\pi}(y) \cdot \gamma, \quad \forall y \in \tilde{Y}, \gamma \in \Gamma .
$$

(ii) The leaves of the foliation $\mathcal{F}$ are given by the images of the fibres of the fibration $\tilde{\pi}: \widetilde{Y} \rightarrow \widetilde{B}$ under the quotient map

$$
q_{\tilde{\pi}}: \tilde{Y} \rightarrow Y
$$

Thus the leaves of the foliation are given by $q_{\widetilde{\pi}}\left(\widetilde{\pi}^{-1}(y)\right)$ for $y \in \widetilde{B}$.

Let $\bar{M}$ be a smooth manifold with boundary $\partial M$ equipped with a Seifert fibration $\mathcal{F}$. Denote by $M$ the interior of $\bar{M}$. Let $B$ be the space of leaves and let $\pi: \partial M \rightarrow B$ be the associated projection. Since $B$ is an orbifold, we know (see for instance [23, $\S 4.4 .10]$ ) that it has a natural induced stratification defined in terms of the isotropy types of the points on $B$, which are defined as follows. If $(\mathcal{U}, \widetilde{\mathcal{U}}, \Gamma, \varphi)$ is an orbifold chart for an open neighborhood $\mathcal{U}$ of a point $p \in B$, then the isotropy type of $p$, denoted $\left[I_{p}\right]$, is the isomorphism class of the isotropy group $I_{\widetilde{p}}$ of $\widetilde{p}$ under the action of $\Gamma$, where $\widetilde{p}$ is any point in $\varphi^{-1}(p)$. As can be readily checked, the isotropy type depends neither on the choice of $\widetilde{p}$ nor on the orbifold chart. In terms of the isotropy type, the stratification of $B$ is given by

$$
B=B_{b} \supset B_{b-1} \supset \cdots \supset B_{1} \supset B_{0}, \quad \text { with } b=\operatorname{dim} B,
$$

where the stratum $B_{i}$ is the disjoint union of all the strata of dimension $i$ of the form

$$
s_{[\Gamma]}=\overline{\left\{p \in B:\left[I_{p}\right]=[\Gamma]\right\}},
$$


where the closure is taken in $B$ and $\left[I_{p}\right]=[\Gamma]$ means that the group $I_{p}$ is isomorphic to the group $\Gamma$. That 10 is a well-defined stratification can be seen locally in an orbifold chart by using the corresponding result about the stratification of manifolds admitting a smooth group action, see for instance [9]. Recall that the depth $\delta\left(s_{[\Gamma]}\right)$ of a stratum $s_{[\Gamma]}$ of $B$ is the biggest integer $k$ such that there exists different strata $s_{0}, \ldots, s_{k-1}$ such that

$$
s_{0} \varsubsetneqq s_{1} \varsubsetneqq \cdots \varsubsetneqq s_{k-1} \varsubsetneqq s_{k}:=s_{[\Gamma]} .
$$

The depth of $B$ is

$$
\delta(B)=\sup \left\{\delta\left(s_{[\Gamma]}\right): s_{[\Gamma]} \text { is a stratum of } B\right\} .
$$

Let $X$ be the singular space given in (4). Then $X$ is naturally a stratified space with stratification

$$
X_{n} \supset X_{n-1} \supset X_{n-2} \supset \cdots \supset X_{1} \supset X_{0}
$$

induced by of $B$, i.e.

$$
X_{j}:= \begin{cases}X, & j=n, \\ B, & b \leq j<n, \\ B_{j}, & j<b\end{cases}
$$

With this stratification, the depth of $X$ is one more than that of $B$, i.e. $\delta(X)=\delta(B)+1$.

We will now discuss regular neighborhoods for points $p \in B \subset X$. We will use the following standard notation for cones; given $\epsilon>0$ and a topological space $Z$, set

$$
C_{\epsilon}(Z):=Z \times[0, \epsilon] / Z \times\{0\} .
$$

Lemma 12. Given $p \in B \subset X$, let $\Gamma$ represent the isotropy type $\left[I_{p}\right]$ of $p$ in $B$. Let $b-d$ be the smallest dimension of a stratum $s_{[\Gamma]}$ in $B$ containing $p$. Then there exists a smooth compact $\Gamma$-manifold $\widetilde{F}$ and a smooth $\Gamma$ action on the unit ball $\mathbb{B}^{d}$ such that the $\Gamma$ action on $\mathbb{B}^{d} \times \widetilde{F}$ is free and $p$ has a regular neighborhood in $X$ of the form

$$
U=\mathbb{B}^{b-d} \times C_{1}(L),
$$

where $L=\widetilde{L} / \Gamma$ and $\widetilde{L}$ is the stratified space, with the obvious induced $\Gamma$ action, obtained from $\mathbb{B}^{d} \times \widetilde{F}$ by collapsing the boundary fibration $\partial \mathbb{B}^{d} \times \widetilde{F} \rightarrow \partial \mathbb{B}^{d}$ onto its base. If $d=0$, then $\widetilde{L}=\widetilde{F}$. See Figure 1 . Furthermore,

$$
\delta(L)<\delta(X) .
$$

Proof. Let $V \subset B$ be an open neighborhood of $p$ and $(V, \widetilde{V}, \Gamma, \varphi)$ an associated orbifold chart. Without loss of generality, we can assume that $V$ and the associated orbifold chart are chosen in such a way that there is a unique $\widetilde{p} \in \widetilde{V}$ such that $\varphi \circ q(\widetilde{p})=p$, where $q: \widetilde{V} \rightarrow \widetilde{V} / \Gamma$ is the quotient map. This means the action of $\Gamma$ on $\widetilde{V}$ fixes $\widetilde{p}$, so that the isotropy type of $p$ is precisely the isomorphism class of the group $\Gamma$. By taking $\widetilde{V}$ even smaller if needed, we know by the tube theorem for smooth Lie group actions (see for instance Theorem 2.4.1 in [9]), that we can assume $\widetilde{V}$ is a linear representation of $\Gamma$. Putting an invariant inner product on $\widetilde{V}$, we thus see there is a decomposition

$$
\widetilde{V}=\widetilde{V}^{\Gamma} \times \widetilde{V}^{\prime}
$$

obtained by taking the orthogonal projection on the subvector space $\widetilde{V}^{\Gamma}$ of points fixed by $\Gamma$. Note that if $d=0$ then $\widetilde{V}=\widetilde{V}^{\prime}$. By Remark 8 and at the cost of taking $V$ even smaller, 
we can assume the Seifert fibration on $\partial M$ restricted to $V$ lifts on $\widetilde{V}$ to a $\Gamma$-equivariant fibration,

$$
\text { pr: } \widetilde{V} \times \widetilde{F} \rightarrow \widetilde{V}
$$

where $\widetilde{F}$ is a smooth compact $\Gamma$-manifold and pr is the projection on the first factor. The smooth action of $\Gamma$ on the total space $\widetilde{V} \times \widetilde{F}$ is free and we thus have a quotient map $q: \widetilde{V} \times \widetilde{F} \rightarrow W$ onto the total space $W \subset \partial M$ of the Seifert fibration lying above $V \subset B$.

Let $\mathbb{B}^{\widetilde{V}^{\Gamma}}$ be the open unit ball in $\widetilde{V}^{\Gamma}$. Let $\mathbb{B}^{\widetilde{V}^{\prime} \times \mathbb{R}_{x}}$ be the unit ball in $\widetilde{V}^{\prime} \times \mathbb{R}_{x}$ and let $\mathbb{B}_{+}^{\widetilde{V}^{\prime} \times \mathbb{R}_{x}}=\mathbb{B}^{\widetilde{V}^{\prime} \times \mathbb{R}_{x}} \cap\{x \geq 0\}$ be the corresponding half-ball. With $\Gamma$ acting trivially on $[0, \epsilon)_{x}$ and assuming without loss of generality that $\epsilon>1$, we see that $\mathbb{B}^{\widetilde{V}^{\Gamma}} \times \mathbb{B}_{+}^{\widetilde{V}^{\prime} \times \mathbb{R}_{x}}$ is an open subset of $\widetilde{V} \times[0, \epsilon)_{x}$ which is preserved by the action of $\Gamma$. Thus, it descends to an open subset in $W \times[0, \epsilon)_{x} \subset \bar{M}$. Under the collapsing map $c_{\pi}: \bar{M} \rightarrow X$, this open subset is mapped to a regular neighborhood $U$ of $p \in X$. To see this, notice that under

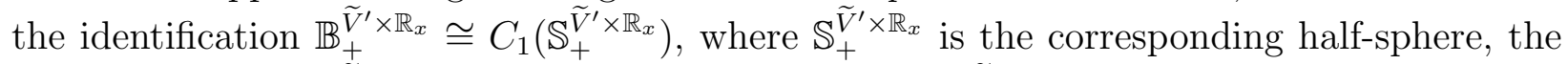

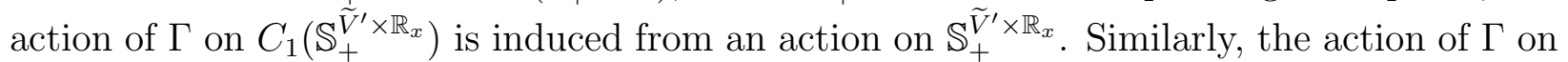


to the stratified space $\widetilde{L}$ given by

$$
\widetilde{L}=\mathbb{S}_{+}^{\widetilde{V}^{\prime} \times \mathbb{R}_{x}} \times \widetilde{F} / \sim
$$

where

$$
\left(s_{1}, f_{1}\right) \sim\left(s_{2}, f_{2}\right) \Longleftrightarrow\left(s_{1}, f_{1}\right)=\left(s_{2}, f_{2}\right) \text { or } s_{1}=s_{2} \in \partial \mathbb{S}_{+}^{\widetilde{V}^{\prime} \times \mathbb{R}_{x}},
$$

see Figure 1. If $L=\widetilde{L} / \Gamma$ is the corresponding stratified space obtained by taking the quotient with respect to this action, then the above identification shows there is a natural homeomorphism

$$
U \cong \widetilde{U}^{\Gamma} \times C_{1}(L) .
$$

If $d=0$, then $\widetilde{V}^{\prime}=\{0\}$ and $\widetilde{L}=\widetilde{F}$. Finally, to establish 12 , note that if $d>0$, then the depth of $L$ is given by

$$
\delta(L)=1+\delta\left(\partial \mathbb{S}_{+}^{\widetilde{V}^{\prime} \times \mathbb{R}_{x}} / \Gamma\right)
$$

and that the depth of $\partial \mathbb{S}_{+}^{V^{\prime}} \times \mathbb{R}_{x} / \Gamma$ is strictly less than the depth of $B$. Thus 12 follows from $\delta(X)=\delta(B)+1$ in this case. If $d=0$, then $L$ is smooth, so $\delta(L)=0<1=\delta(X)$ and (12) follows again.

Definition 13. We will refer to the space $\widetilde{U}=\mathbb{B}^{b-d} \times C_{1}(\widetilde{L})$ with its natural action of $\Gamma$ and quotient map $q: \widetilde{U} \longrightarrow U$ as a resolution of $U$.

\section{From intersection COHOMOlogy to Weighted COHOMOlOGy}

We will now relate the intersection cohomology of $X$ to the cohomology of a complex of sheaves of weighted $L^{2}$ differential forms. The proof will use sheaf theory and will be local on the space of leaves of the boundary foliation. For this reason, we do not yet require that the Seifert fibration on the boundary be good. The main ingredient reducing the discussion to local considerations is the following fundamental result from [13, p.104], cf. Proposition 1 in $[14$. 


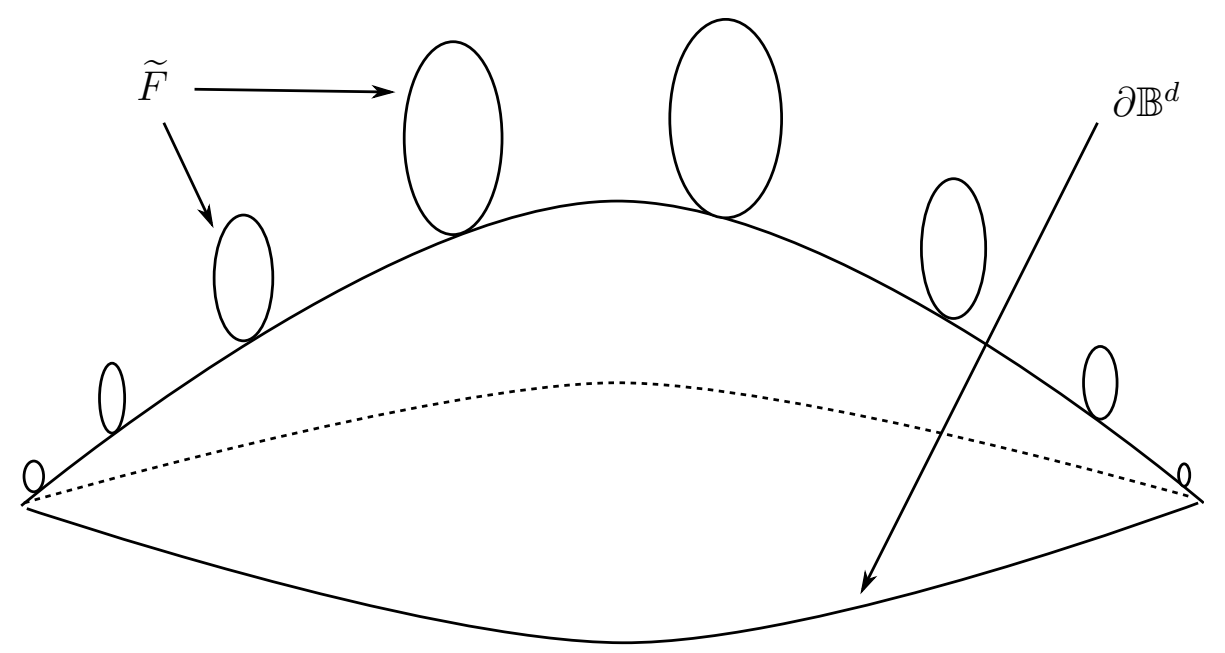

Figure 1. the space $\widetilde{L}$ from $(13)$, where $\mathbb{S}_{+}^{\widetilde{V}^{\prime} \times \mathbb{R}_{x}}$ is written simply as $\mathbb{B}^{d}$

Proposition 14 ([13]). Let $X$ be a stratified space, and let $\left(\mathcal{L}^{*}, d\right)$ be a complex of fine sheaves on $X$ with cohomology $H^{*}(X, \mathcal{L})$. Suppose that if $U$ is a neighborhood in the principal (smooth) stratum of $X$, then $H^{*}(U, \mathcal{L})=H^{*}(U, \mathbb{C})$, while if $q$ lies in a stratum of codimension $\ell$ and $U=V \times C_{1}(L)$ as in (11), then

$$
H^{*}(U, \mathcal{L}) \simeq I H_{\mathfrak{p}}^{k}(U)=\left\{\begin{array}{cl}
I H_{\mathfrak{p}}^{k}(L), & k \leq \ell-2-\mathfrak{p}(\ell) \\
0, & k \geq \ell-1-\mathfrak{p}(\ell) .
\end{array}\right.
$$

Then there is a natural isomorphism between the hypercohomology $\mathbb{H}^{*}\left(X, \mathcal{L}^{*}\right)$ associated to this complex of sheaves and $I H_{\mathfrak{p}}^{*}(X)$, the intersection cohomology with perversity $\mathfrak{p}$.

Before we continue, we take a moment to give the general definition of foliated boundary and foliated cusp metrics, following [24]. Foliated boundary metrics are metrics on a vector bundle over the compact manifold with boundary $\bar{M}$, defined as follows. First, for a fixed boundary defining function $x$ and a choice of foliation $\mathcal{F}$ on $\partial \bar{M}$, we have the space of foliated cusp vector fields

$$
\mathcal{V}_{\mathcal{F}}(M)=\left\{\xi \in \Gamma(\mathrm{T} \bar{M}): \xi x=x^{2} C^{\infty}(\bar{M}) \text { and }\left.\xi\right|_{\partial M} \in \Gamma(\mathrm{T} \mathcal{F})\right\} .
$$

This set of vector fields over $\bar{M}$ is a Lie algebra under Lie bracket. It can be identified with the space of smooth sections of the vector bundle ${ }^{\mathcal{F}} T \bar{M}$, whose fibre at each point $p \in \bar{M}$ is the set $\mathcal{V}_{\mathcal{F}}(M) /\left(\mathcal{I}_{p}(\bar{M}) \mathcal{V}_{\mathcal{F}}(M)\right)$, where $\mathcal{I}_{p}(\bar{M}) \subset C^{\infty}(\bar{M})$ is the ideal of smooth functions vanishing at $p$. Then a foliated boundary metric is a Riemannian metric $g_{\mathcal{F}}$ on $M$ induced by the identification $T M=\left.{ }^{\mathcal{F}} T \bar{M}\right|_{M}$ and a choice of a Euclidean metric on the bundle ${ }^{\mathcal{F}} T \bar{M}$. A foliated cusp metric is a Riemannian metric on $M$ of the form $g_{\mathcal{F}-c}=x^{2} g_{\mathcal{F}}$ for some foliated boundary metric $g_{\mathcal{F}}$ on $M$. When $\mathcal{F}$ is a Seifert fibration, examples of such metrics are given by the exact foliated boundary and exact foliated cusp metrics described in the introduction.

Given an open set $U \subset X$, Let $L^{2} \Omega^{k}\left(g_{\mathcal{F}-c}, U\right)$ denote the completion of the space of compactly supported differential $k$-forms on $U-B$ with respect to the $L^{2}$-norm induced by $g_{\mathcal{F}-c}$, and let $x^{a} L^{2} \Omega^{k}\left(g_{\mathcal{F}-c}, U\right)$ denote the space of currents $\omega$ satisfying $x^{-a} \omega \in L^{2} \Omega^{k}\left(g_{\mathcal{F}-c}, U\right)$. By considering the subspace

$$
x^{a} L_{d}^{2} \Omega^{k}\left(g_{\mathcal{F}-c}, U\right):=\left\{\omega \in x^{a} L^{2} \Omega^{k}\left(g_{\mathcal{F}-c}, U\right) d \omega \in x^{a} L^{2} \Omega^{k+1}\left(g_{\mathcal{F}-c}, U\right)\right\},
$$


we obtain a complex

$$
\cdots \stackrel{d}{\rightarrow} x^{a} L_{d}^{2} \Omega^{k}\left(g_{\mathcal{F}-c}, U\right) \stackrel{d}{\rightarrow} x^{a} L_{d}^{2} \Omega^{k+1}\left(g_{\mathcal{F}-c}, U\right) \stackrel{d}{\rightarrow} \cdots .
$$

The corresponding cohomology groups are given by

$$
W H^{k}\left(g_{\mathcal{F}-c}, a, U\right)=\frac{\left\{\omega \in x^{a} L^{2} \Omega^{k}\left(g_{\mathcal{F}-c}, U\right): d \omega=0\right\}}{\left\{d \zeta \in x^{a} L^{2} \Omega^{k}\left(g_{\mathcal{F}-c}, U\right): \zeta \in x^{a} L^{2} \Omega^{k-1}\left(g_{\mathcal{F}-c}, U\right)\right\}} .
$$

For a foliated boundary metric, $g_{\mathcal{F}}$, we consider instead the complex

$$
\cdots \stackrel{d}{\rightarrow} x^{a-1} L_{d}^{2} \Omega^{k-1}\left(g_{\mathcal{F}}, U\right) \stackrel{d}{\rightarrow} x^{a} L_{d}^{2} \Omega^{k}\left(g_{\mathcal{F}}, U\right) \stackrel{d}{\rightarrow} x^{a+1} L_{d}^{2} \Omega^{k+1}\left(g_{\mathcal{F}}, U\right) \stackrel{d}{\rightarrow} \cdots,
$$

where this time

$$
x^{a} L_{d}^{2} \Omega^{k}\left(g_{\mathcal{F}}, U\right)=\left\{\omega \in x^{a} L^{2} \Omega^{k}\left(g_{\mathcal{F}}, U\right): d \omega \in x^{a+1} L^{2} \Omega^{k+1}\left(g_{\mathcal{F}}, U\right)\right\} .
$$

The corresponding cohomology groups are then denoted by

$$
\mathcal{W} H^{k}\left(g_{\mathcal{F}}, a, U\right):=\frac{\left\{\omega \in x^{a} L^{2} \Omega^{k}\left(g_{\mathcal{F}}, U\right): d \omega=0\right\}}{\left\{d \zeta \in x^{a} L^{2} \Omega^{k}\left(g_{\mathcal{F}}, U\right): \zeta \in x^{a-1} L^{2} \Omega^{k-1}\left(g_{\mathcal{F}}, U\right)\right\}} .
$$

For a fixed boundary foliation $\mathcal{F}$ and a fixed boundary defining function $x \in \mathcal{C}^{\infty}(\bar{M})$, the space $x^{a} L_{d}^{2} \Omega^{k}\left(g_{\mathcal{F}}, U\right)$ and thus the group $\mathcal{W} H^{k}\left(g_{\mathcal{F}}, a, U\right)$ do not depend on the choice of the foliated boundary metric $g_{\mathcal{F}}$. Indeed, if $g_{\mathcal{F}}$ and $g_{\mathcal{F}}^{\prime}$ are two foliated boundary metrics, then by the compactness of $\bar{M}$ there exists a constant $C>0$ such that

$$
\frac{g_{\mathcal{F}}}{C} \leq g_{\mathcal{F}}^{\prime} \leq C g_{\mathcal{F}}
$$

so the weighted $L^{2}$ spaces, and thus the cohomologies, are the same for either metric. The same invariance holds for foliated cusp metrics. In particular, we may assume that $x^{2} g_{\mathcal{F}}=$ $g_{\mathcal{F}-c}$. It follows that $x^{a} L^{2} \Omega^{k}\left(g_{\mathcal{F}}, U\right)=x^{n / 2-k+a} L^{2} \Omega^{k}\left(g_{\mathcal{F}-c}, U\right)$, and consequently that

$$
\mathcal{W} H^{k}\left(g_{\mathcal{F}}, a, U\right)=W H^{k}\left(g_{\mathcal{F}-c},(n / 2)-k+a, U\right) .
$$

Because we will work predominantly with fibred cusp metrics in this section, and because the particular choice of fibred cusp metric does not effect the weighted cohomology, we will often write simply

$$
W H^{k}(a, U):=W H^{k}\left(g_{\mathcal{F}-c}, a, U\right) .
$$

As explained in Section 2.1 of [14] using the Kodaira decomposition theorem, the same invariance under quasi-isometries holds for Hodge cohomology. For instance, for any two foliated cusp metrics $g_{\mathcal{F}-c}$ and $g_{\mathcal{F}-c}^{\prime}$ associated to the same choices of foliation $\mathcal{F}$ and boundary defining function $x, L^{2} \mathcal{H}^{k}\left(M, g_{\mathcal{F}-c}^{\prime}\right) \cong L^{2} \mathcal{H}^{k}\left(M, g_{\mathcal{F}-c}\right)$.

The purpose of this section is to prove the following analogue of Proposition 2 in [14].

Theorem 15. Let $M$ be a manifold with boundary foliated by a Seifert fibration $\mathcal{F}$, and let $g_{\mathcal{F}-c}$ be an associated foliated cusp metric on $M$. Suppose that $k-1+a-f / 2 \neq 0$ whenever $H^{k}(\widetilde{F}) \neq 0$. Then

$$
W H^{*}\left(g_{\mathcal{F}-c}, a, M\right) \simeq I H_{[a+f / 2]}^{*}(X, B),
$$

where $[a+f / 2]$ is the greatest integer less than or equal to $a+f / 2$.

We notice two easy consequences of the theorem. 
Corollary 16. If $k-1+a-f / 2 \neq 0$ whenever $H^{k}(\widetilde{F}) \neq 0$, then the differential in the complex (15) has closed range.

Proof. By the theorem, $W H^{*}\left(g_{\mathcal{F}-c}, a, M\right)$ is finite dimensional, so the differential must have closed range.

Corollary 17. The intersection cohomology $I_{\mathfrak{p}}^{*}(X)$ depends only on $\mathfrak{p}(f+1)$.

Proof. Use the identification (19) with $a \in \mathbb{R}$ such that $2 a \notin \mathbb{Z}$ and $\left[a+\frac{f}{2}\right]=\mathfrak{p}(f+1)$.

The main tool in the proof of Theorem 15 is Proposition 14. To apply this proposition, we must know that the sheaf which associates to each open set $U \subset X$ the complex in (15) is fine. Indeed, given an open cover $\left\{O_{k}\right\}_{k \in K}$, we can choose a subcover $\left\{U_{i}\right\}_{i \in I}$ so that each $U_{i}$ is a regular neighborhood as in (11) with a resolution $q: \widetilde{U}_{i} \longrightarrow U_{i}$. Let $\left\{V_{i}\right\}_{i \in I}$ be yet another subcover so that $\overline{V_{i}} \subset U_{i}$. Let $\widetilde{V}_{i}=q^{-1}\left(V_{i}\right)$. If $U_{i}$ is supported away from $B$, let $\rho_{i} \in \mathcal{C}^{\infty}\left(U_{i}\right)$ be a nonnegative function with compact support equal to 1 on $V_{i}$. On the other hand, if $U_{i}$ intersects $B$, we are assuming that $\widetilde{U}_{i}$ is of the form $\widetilde{U}_{i}=\mathbb{B}^{b} \times C_{\epsilon}(\widetilde{F})$. Thus, there is a projection $\widetilde{\pi}: \widetilde{U}_{i} \rightarrow \mathbb{B}^{b} \times[0, \epsilon)_{x}$ given by

$$
\tilde{\pi}(b, x, f)=(b, x) .
$$

Let $\phi_{i} \in \mathcal{C}^{\infty}\left(\mathbb{B}^{b} \times[0, \epsilon)_{x}\right)$ be a nonnegative smooth function with compact support such that $\tilde{\pi}^{*} \phi_{i}$ is equal to 1 on $\widetilde{V}_{i}$ and $\phi_{i}$ is constant near $x=0$. Clearly, since $\tilde{\pi}^{*} \phi_{i}$ is constant along $\widetilde{F}$, the norm of $d\left(\widetilde{\pi}^{*} \phi_{i}\right)$ is bounded with respect to a choice of fibred cusp metric on $\widetilde{U}_{i}$. Averaging with respect to $\Gamma$, we can assume $\phi_{i}$ is $\Gamma$-invariant. Thus, the function $\widetilde{\pi}^{*} \phi_{i}$ descends to a smooth function $\rho_{i}$ with compact support on $U_{i}$. As usual, the functions $\chi_{i}:=\rho_{i} / \sum \rho_{j}$ form a partition of unity subordinate to the cover $\left\{O_{k}\right\}_{k \in K}$. The fact that the differential $d \chi_{i}$ has norm bounded with respect to a choice of foliated cusp metric insures that it acts by multiplication on $x^{a} L_{d}^{2} \Omega^{k}\left(g_{\mathcal{F}-c}, U_{i}\right)$. Thus, the associated sheaf is fine.

As a preliminary to the proof of Theorem 15, we discuss the computation of the weighted cohomology of $C_{\epsilon}(\widetilde{F})$ from Proposition 2 of [14]. There, they show that, provided

$$
k-1+a-f / 2 \neq 0 \quad \text { whenever } \quad H^{k}(\widetilde{F}) \neq 0,
$$

we have

$$
W H^{k}\left(a, C_{\epsilon}(\widetilde{F})\right) \simeq\left\{\begin{array}{cl}
H^{k}(\widetilde{F}) & \text { if } k<f / 2-a \\
0 & \text { if } k \geq f / 2-a .
\end{array}\right.
$$

If $\iota: \widetilde{F} \longrightarrow C_{\epsilon}(\widetilde{F})$ is the inclusion of $\widetilde{F}$ into $\widetilde{F} \times\{\epsilon\}$, then the isomorphism with $H^{k}(\widetilde{F})$ in the case $k<f / 2-a$ is induced by $\iota$. Since we will need the proof in a moment, we recall it now but direct the reader to [14] for more details. By the $L^{2}$-Künneth theorem from [31], as long as 20 holds, $W H^{k}\left(a, C_{\epsilon}(\widetilde{F})\right)$ is isomorphic to

$$
\begin{aligned}
& \left(W H^{0}\left((0, \epsilon), d x^{2} / x^{2}, k+a-f / 2\right) \otimes H^{k}(\widetilde{F})\right) \oplus \\
& \left(W H^{1}\left((0, \epsilon), d x^{2} / x^{2}, k-1+a-f / 2\right) \otimes H^{k-1}(\widetilde{F})\right) .
\end{aligned}
$$

It is trivial to check that $W H^{1}\left((0, \epsilon), d x^{2} / x^{2}, b\right)=0$ if $b \neq 0$, and that $W H^{0}\left((0, \epsilon), d x^{2} / x^{2}, b\right)$ is 0 for $b \geq 0$ and $\mathbb{C}$ for $b<0$. Combining these two facts with (22) proves (21), and the naturality of the Künneth formula shows that the isomorphism is induced by the inclusion $\iota$. 
For the space $\widetilde{L}$ in Lemma 12 , if $d=1$ we can use the exact same argument to compute its weighted cohomology. In this case, $\widetilde{L}=[0,1]_{x} \times \widetilde{F} /(\{0\} \times \widetilde{F} \cup\{1\} \times \widetilde{F})$, and by invariance, we can take the fibred cusp metric on $\widetilde{L}$ to be

$$
g=f^{-1}(x) d x^{2}+f(x) \widetilde{k},
$$

where $f$ is a smooth positive function on $(0,1)$ with

$$
f(x)=\left\{\begin{array}{ccc}
x^{2} & \text { for } & x<1 / 4 \\
(x-1)^{2} & \text { for } & x>3 / 4
\end{array}\right.
$$

and $\widetilde{k}$ is a metric on $\widetilde{F}$. An argument identical to that of the previous paragraph yields (21) with $C_{\epsilon}(\widetilde{F})$ replaced by $\widetilde{L}$ in $(21)$ and $d x^{2} / x^{2}$ replaced by $d x^{2} / f$ in (22). In fact, if for small $\epsilon>0$, we consider $C_{\epsilon}(\widetilde{F}) \stackrel{\iota}{\rightarrow} \widetilde{L}$, where $\iota(x, f)=(x, f)$, then we have shown that

$$
\iota^{*}: W H^{k}(a, \widetilde{L}) \longrightarrow W H^{k}\left(a, C_{\epsilon}(\widetilde{F})\right)
$$

is an isomorphism (provided (20) holds).

To obtain a similar result for $d>1$, consider the following sequence of inclusions

$$
C_{\epsilon}(\widetilde{F}) \stackrel{\iota}{\longrightarrow} \widetilde{L} \stackrel{j}{\longrightarrow} C_{1}(\widetilde{L})
$$

where $\iota$ is defined as follows. Near the boundary of $\mathbb{B}^{d}$, let $(x, y)$ be coordinates where $x$ is a b.d.f. and $y$ is a coordinate on the sphere. Then set

$$
\iota(x, f):=(x, y, f) \text { for some fixed but arbitrary } y \text {. }
$$

We endow $\widetilde{L}$ with a foliated cusp metric by considering the foliated cusp metric $g_{\mathcal{F}-c}$ of $X$ on a canonical neighborhood $U \simeq \mathbb{B}^{b-d} \times C_{1}(L)$ as in (11), pulling $g_{\mathcal{F}-c}$ to $\left\{p_{0}\right\} \times C_{1}(L)$ for a fixed $p_{0} \in \mathbb{B}^{b-d}$, i.e. to the inclusion of a cone $C_{1}(L)$ over an arbitrary point in $\mathbb{B}^{b-d}$, and then lifting to $C_{1}(\widetilde{L})$. Finally, endow $C_{\epsilon}(\widetilde{F})$ with the pullback metric induced by $j \circ \iota$ in 26 ).

Lemma 18. If $k-1+a-\frac{f}{2} \neq 0$ whenever $H^{k}(\widetilde{F}) \neq 0$, then the induced mappings

$$
\begin{aligned}
\iota^{*}: W H^{k}(a, \widetilde{L}) & \longrightarrow W H^{k}\left(a, C_{\epsilon}(\widetilde{F})\right) \\
j^{*}: W H^{k}\left(a, C_{1}(\widetilde{L})\right) & \longrightarrow W H^{k}(a, \widetilde{L}) .
\end{aligned}
$$

are isomorphisms.

Proof. To see that $\iota^{*}$ is an isomorphism, we will proceed by induction on $d,(25)$ being the base case. Assume the result holds for $d \leq m-1$. We need to show the result holds for $d=m$. Let $\left(y^{1}, \ldots, y^{d}\right)$ denote the standard coordinates on $\mathbb{R}^{d}$ and consider the open sets in $\mathbb{B}^{d}$ given by

$$
\begin{gathered}
U=\left\{y=\left(y^{1}, \ldots, y^{d}\right) \in \mathbb{B}^{d}: y^{1}<1 / 2\right\} \\
V=\left\{y=\left(y^{1}, \ldots, y^{d}\right) \in \mathbb{B}^{d}: y^{1}>-1 / 2\right\} .
\end{gathered}
$$


Let $\widetilde{U}=\operatorname{pr}^{-1}(U)$ and $\widetilde{V}=\operatorname{pr}^{-1}(V)$ be the corresponding open sets in $\widetilde{L}$, where $\operatorname{pr}: \widetilde{L}=$ $\mathbb{B}^{d} \times \widetilde{F} / \sim \rightarrow \mathbb{B}^{d}$ is the natural projection. Since $\widetilde{L}=\widetilde{U} \cup \widetilde{V}$, there is an induced MayerVietoris sequence

$$
\begin{aligned}
\cdots & \stackrel{\widetilde{\Delta}}{\rightarrow} W H^{k-1}(a, \widetilde{U} \cap \widetilde{V}) \stackrel{\widetilde{\delta}}{\rightarrow} W H^{k}(a, \widetilde{L}) \stackrel{\widetilde{\psi}}{\rightarrow} \\
& W H^{k}(a, \widetilde{U}) \oplus W H^{k}(a, \widetilde{V}) \stackrel{\widetilde{\Delta}}{\rightarrow} W H^{k}(a, \widetilde{U} \cap \widetilde{V}) \stackrel{\widetilde{\delta}}{\rightarrow} \cdots
\end{aligned}
$$

Since $\mathbb{B}^{d-1} \times \widetilde{F} / \sim$ is a deformation retract of $\widetilde{U} \cap \widetilde{V}$ through maps which preserve the stratification, we see by our induction hypothesis that

$$
W H^{k}(a, \widetilde{U} \cap \widetilde{V}) \simeq W H^{k}\left(a, C_{\epsilon}(\widetilde{F})\right) .
$$

Since $\widetilde{U}$ and $\widetilde{V}$ both retract onto $C_{\epsilon}(\widetilde{F})$ (again, preserving the stratification), we also have canonical identifications

$$
W H^{k}(a, \widetilde{U})=W H^{k}\left(a, C_{\epsilon}(\widetilde{F})\right), \quad W H^{k}(a, \widetilde{V})=W H^{k}\left(a, C_{\epsilon}(\widetilde{F})\right) .
$$

In terms of these identifications, the map $\widetilde{\Delta}$ is given by

$$
\begin{aligned}
\widetilde{\Delta}: W H^{k}\left(a, C_{\epsilon}(\widetilde{F})\right) \oplus W H^{k}\left(a, C_{\epsilon}(\widetilde{F})\right) & \rightarrow W H^{k}\left(a, C_{\epsilon}(\widetilde{F})\right) \\
\left(\omega_{1}, \omega_{2}\right) & \mapsto \omega_{1}-\omega_{2}
\end{aligned}
$$

and is surjective. The boundary homomorphism $\widetilde{\delta}$ is therefore trivial and the map $\widetilde{\psi}$ gives an isomorphism

$$
\widetilde{\psi}: W H^{k}(a, \widetilde{L}) \rightarrow \operatorname{ker} \widetilde{\Delta} \cong W H^{k}\left(a, C_{\epsilon}(\widetilde{F})\right) \text {. }
$$

Since $\widetilde{\psi}$ corresponds to the map $\iota^{*}$ under the identification $\operatorname{ker} \widetilde{\Delta} \cong W H^{k}\left(a, C_{\epsilon}(\widetilde{F})\right)$, we see $\iota^{*}$ induces an isomorphism in weighted cohomology.

To see that $j^{*}$ induces an isomorphism, notice that since $C_{\epsilon}(\widetilde{F})$ is a deformation retract of $C_{1}(\widetilde{L})$, we easily see that

$$
\iota^{*} \circ j^{*}: W H^{k}\left(a, C_{1}(\widetilde{L})\right) \longrightarrow W H^{k}\left(a, C_{\epsilon}(\widetilde{F})\right)
$$

is an isomorphism, and thus that $j^{*}=\left(\iota^{*}\right)^{-1} \circ\left(\iota^{*} \circ j^{*}\right)$ is an isomorphism as well.

Proof of Theorem 15. We proceed by induction on the depth of $X$, the base case being that of a smooth manifold, where the theorem is trivial. Assume that Theorem 15 is proven for foliated cusp metrics of depth not greater than $\delta-1$ and that $\delta(X)=\delta$.

Let $q \in X_{n-j+1}-X_{n-j}$ and choose a regular neighborhood as described in Lemma 12 , $U \simeq V \times C_{1}(L)$. By Proposition 14, we need to show that $W H^{k}(a, U) \simeq W H^{k}\left(a, C_{1}(L)\right)$ is isomorphic to $I H_{[a+f / 2]}^{k}(U) \simeq I H_{[a+f / 2]}^{k}\left(C_{1}(L)\right)$. In a moment, we will show that

$$
I H_{[a+f / 2]}^{k}\left(C_{1}(L)\right) \cong I H_{[a+f / 2]}^{k}(L) .
$$

Since the depth of $L$ is smaller than that of $X$, we have by our induction hypothesis that

$$
I H_{[a+f / 2]}^{k}(L) \cong W H^{k}(a, L) .
$$


Thus, it suffices, thanks to (31), to show that the inclusion $i: L \rightarrow C_{1}(L)$ induces an isomorphism in weighted cohomology. To see this, consider the commutative diagram

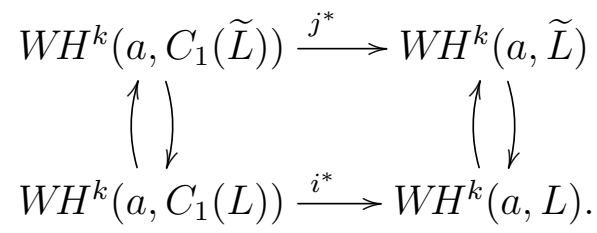

The upward and downward pointing maps are, respectively, the pullback via the projection and the averaging map, and they are injective and surjective, respectively. In fact, the images of the upward pointing maps are exactly the $\Gamma$-invariant elements of the cohomology (i.e. those which can be represented by $\Gamma$-invariant forms.) By Lemma 18 , we know that $j^{*}$ is an isomorphism, and it obviously identifies the $\Gamma$-invariant elements in the two groups. Since these are exactly the images of the upward pointing maps, commutativity shows that $i^{*}$ is an isomorphism as well and the theorem holds for $X$.

To see that (31) holds, note that using (32), (33), and Lemma 18, we have

$$
I H_{[a+f / 2]}^{k}(L) \simeq W H^{k}(a, L) \hookrightarrow W H^{k}(a, \widetilde{L}) \simeq W H^{k}\left(a, C_{\epsilon}(\widetilde{F})\right) .
$$

In degree $k \geq \ell-1-p(\ell)$, the latter is zero by (21) and

$$
p(\ell) \leq p(f+1)+\ell-(f+1) \Longrightarrow \ell-1-p(\ell) \geq-a+f / 2,
$$

so (31) follows from (14) in Proposition 14 .

Using a similar approach by induction on the depth together with Proposition 14, one can show the following result about the intersection cohomology of orbifolds. This is well-known, but since we were unable to find a reference, we include a proof for completeness.

Theorem 19. The intersection cohomology of a compact orbifold $B$ is independent of the choice of standard perversity $\mathfrak{p}$ and is given by singular cohomology (with real coefficients),

$$
I H_{\mathfrak{p}}^{*}(B) \cong H^{k}(B ; \mathbb{R})
$$

Proof. Really, we will show intersection cohomology is given by the (orbifold) de Rham cohomology, which is identified with the singular cohomology (with real coefficients). We will proceed by induction on the depth of the orbifold. For an orbifold of depth 0, the result is trivial. Thus, assume the result is true for compact orbifolds of depth less than $\delta$ and suppose $B$ is a compact orbifold of depth $\delta$. Let $\Omega^{*}$ be the complex of sheaves of smooth orbifold forms on $B$. For a point $b \in B$, we can find a neighborhood of the form $U=\mathbb{B}^{k} \times C_{1}(L)$, where $L$ is an orbifold of depth less than the one of $B$ obtained by taking the quotient of the sphere $\mathbb{S}^{\ell-1}$ by the smooth action of some finite group $\Gamma, L=\mathbb{S}^{\ell-1} / \Gamma$.

Since $U$ is contractible, we have that

$$
H^{k}\left(\Omega^{*}(U)\right)= \begin{cases}\mathbb{R} ; & k=0, \\ 0, & k>0 .\end{cases}
$$

On the other hand, by our induction hypothesis, the result holds on $L=\mathbb{S}^{\ell-1} / \Gamma$, so that

$$
I H_{\mathfrak{p}}^{k}(L)=H^{k}\left(\Omega^{*}(L)\right)= \begin{cases}\mathbb{R} ; & k=0, \ell-1, \\ 0, & \text { otherwise }\end{cases}
$$


Since

$$
\mathfrak{p}(\ell) \geq 0 \Longrightarrow \ell-1-\mathfrak{p}(\ell) \leq \ell-1,
$$

we thus see that we can rewrite $(34)$ as

$$
H^{k}\left(\Omega^{*}(U)\right)= \begin{cases}I H_{\mathfrak{p}}^{k}(L) ; & k \leq \ell-2-\mathfrak{p}(\ell), \\ 0, & k \geq \ell-1-\mathfrak{p}(\ell) .\end{cases}
$$

Since $b \in B$ was arbitrary, we conclude by Proposition 14 that $H^{k}\left(\Omega^{*}(B)\right)=I H_{\mathfrak{p}}^{k}(B)$ for all $k$, completing the inductive step.

Notice in particular that this implies the following known fact about orbifolds, cf. [7].

Corollary 20. Poincaré duality holds for the singular cohomology with real coefficients of a compact oriented orbifold.

\section{From Weighted Cohomology to $L^{2}$ COHomology.}

In this section, we will prove that $L^{2}$ harmonic forms for foliated cusp (and foliated boundary) metrics is isomorphic to the images of maps of appropriately weighted cohomology groups. The main technical tool in the proof is a parametrix for the Hodge-deRham operator on differential forms, for which we rely on the thesis of Vaillant [28]. Since the thesis is lengthy and technical, we will use the description of the parametrix construction in 14 as a guide, and in doing to it will become clear why we need the assumption that $\mathcal{F}$ is a good Seifert fibration as in Definition 11 for the purposes of this section.

Let $D_{\mathcal{F}}=d+\delta_{\mathcal{F}}$ be the Hodge-deRham operator associated to an exact foliated boundary metric $g_{\mathcal{F}}$ on $M$. Let also $D_{\mathcal{F}-c}$ denote the Hodge-deRham operator associated to the conformally related exact foliated cusp metric $g_{\mathcal{F}-c}=x^{2} g_{\mathcal{F}}$. To construct parametrices for these operators, we will make use of their restrictions to the tubular neighborhood $\partial M \times$ $[0, \epsilon)_{x}$. Denote by $\widetilde{D}_{\mathcal{F}}:=q^{*} D_{\mathcal{F}}$ and $\widetilde{D}_{\mathcal{F}-c}:=q^{*} D_{\mathcal{F}-c}$ the pull-back of these restrictions under the quotient map $q: \partial \widetilde{M} \times[0, \epsilon)_{x} \rightarrow \partial M \times[0, \epsilon)_{x}$. Clearly, $\widetilde{D}_{\mathcal{F}}$ and $\widetilde{D}_{\mathcal{F}-c}$ are the Hodge-deRham operators associated to the pull-back metrics $q^{*} g_{\mathcal{F}}$ and $q^{*} g_{\mathcal{F}-c}$. They are $\Gamma$-invariant. This suggests the parametrix construction of [14] (see also [28]) can be used to obtain a parametrix for $D_{\mathcal{F}}$ and $D_{\mathcal{F}-c}$.

We must first introduce some notation. All of the various spaces of differential forms in [14 have analogues on spaces with foliated boundaries, which can be defined by lifting via $q$. For example, we define the space $\Omega_{\mathcal{F}}^{*}(M)$ to be the space of smooth forms on $M$ which, when restricted to $\partial M \times\left(0, \frac{\epsilon}{2}\right)$, pull back via $q_{\widetilde{\pi}}$ to lie in the space $\Omega_{f b}^{*}\left(\partial \widetilde{M} \times[0, \epsilon)_{x}\right)$ in Section 5.1 of [14]. The same goes for the spaces of conormal distributions of degree $k, \mathcal{A}^{*} \Omega_{\mathcal{F}}^{k}(M)$ and polyhomogeneous forms $\mathcal{A}_{p h g}^{*} \Omega_{\mathcal{F}}^{k}(M)$. We then have, for example, the complexes

$$
\cdots \stackrel{d}{\rightarrow} x^{a} \mathcal{A}^{*} \Omega^{k}\left(g_{\mathcal{F}}, U\right) \stackrel{d}{\rightarrow} x^{a+1} \mathcal{A}^{*} \Omega^{k+1}\left(g_{\mathcal{F}}, U\right) \stackrel{d}{\rightarrow} \cdots .
$$

and

$$
\cdots \stackrel{d}{\rightarrow} x^{a} \mathcal{A}^{*} \Omega^{k}\left(g_{\mathcal{F}-c}, U\right) \stackrel{d}{\rightarrow} x^{a} \mathcal{A}^{*} \Omega^{k+1}\left(g_{\mathcal{F}-c}, U\right) \stackrel{d}{\rightarrow} \cdots,
$$

and as described in section 2.5 of [14], the cohomologies of these two complexes are naturally isomorphic to the corresponding weighted $L^{2}$ cohomologies in (15) and (17). The same is true for the polyhomogeneous spaces. 
Let $\Pi_{0}$ be the family of projections onto the space of harmonic forms for the family of Hodge-deRham operators $D_{k}$ associated to the family of metrics $k$ on the fibres of $\widetilde{\pi}: \partial \widetilde{M} \rightarrow$ $B$. Let $\Pi_{\perp}$ be the orthogonal complement of this family.

Definition 21. We define $x^{a+1} \Pi_{0} L^{2} \Omega_{\mathcal{F}}^{*}(M) \oplus x^{a} \Pi_{\perp} L^{2} \Omega_{\mathcal{F}}^{*}(M)$ to be the space of currents on $M$ which are in $L^{2}$ when restricted to $M \backslash\left(\partial M \times\left[0, \frac{\epsilon}{2}\right)\right)$ and are such that their restrictions to $\partial M \times[0, \epsilon)_{x}$ pull back under the quotient map $q_{\tilde{\pi}}$ to give elements in the space $x^{a+1} \Pi_{0} L^{2} \Omega_{f b}^{*}\left(\partial \widetilde{M} \times[0, \epsilon)_{x}\right) \oplus x^{a} \Pi_{\perp} L^{2} \Omega_{f b}^{*}\left(\partial \widetilde{M} \times[0, \epsilon)_{x}\right)$ defined in [14]. The spaces

$$
\begin{gathered}
x^{a-1} \Pi_{0} H^{1} \Omega_{\mathcal{F}}^{*}(M) \oplus x^{a} \Pi_{\perp} H^{1} \Omega_{\mathcal{F}}^{*}(M), \\
x^{a} \Pi_{0} L^{2} \Omega_{\mathcal{F}-c}^{*}(M) \oplus x^{a-1} \Pi_{\perp} L^{2} \Omega_{\mathcal{F}-c}^{*}(M) \text { and } \\
x^{a} \Pi_{0} H^{1} \Omega_{\mathcal{F}-c}^{*}(M) \oplus x^{a+1} \Pi_{\perp} H^{1} \Omega_{\mathcal{F}}^{*}(M)
\end{gathered}
$$

are defined in a similar way.

In order to use the work in Section 5 of [14] directly, we must make one additional assumption, namely that the pullback $q^{*} g_{\mathcal{F}-c}$ is exact, i.e. of the form (3) from the introduction, and that for fixed $x=\epsilon$, the fibre metric $k$ annihilates the orthocomplement of $\mathrm{T} \widetilde{F} \subset\left(\mathrm{T} \partial \widetilde{M},\left.g_{\mathcal{F}-c}\right|_{x=\epsilon}\right)$. As described in [24, $\left.\S 8\right]$, such a metric is easily constructed.

Proposition 22. Suppose that a is not an indicial root for $\Pi_{0} x^{-1} \widetilde{D}_{\mathcal{F}} \Pi_{0}$. Then

$$
D_{\mathcal{F}}: x^{a} H_{\mathcal{F}}^{1}(M) \rightarrow x^{a+1} \Pi_{0} L^{2} \Omega_{\mathcal{F}}^{*}(M) \oplus x^{a} \Pi_{\perp} L^{2} \Omega_{\mathcal{F}}^{*}(M)
$$

and

$$
D_{\mathcal{F}}: x^{a-1} \Pi_{0} H^{1} \Omega_{\mathcal{F}}^{*}(M) \oplus x^{a} \Pi_{\perp} H^{1} \Omega_{\mathcal{F}}^{*}(M) \rightarrow x^{a} L^{2} \Omega_{\mathcal{F}}^{*}(M)
$$

are Fredholm. If $D_{\mathcal{F}} \omega=0$, then $\omega$ is polyhomogeneous with exponents in its expansion determined by the indicial roots of $\Pi_{0} x^{-1} \widetilde{D}_{\mathcal{F}} \Pi_{0}$, while if $\eta \in \mathcal{A}^{a} \Omega_{\mathcal{F}}^{*}(M), \zeta \in x^{c-1} \Pi_{0} H^{1} \Omega_{\mathcal{F}}^{*} \oplus$ $x^{c} \Pi_{\perp} H^{1} \Omega_{\mathcal{F}}^{*}(M)$ for $c<a$ and $\eta=D_{\mathcal{F}} \zeta$, then $\zeta \in \Pi_{0} \mathcal{A}_{\mathrm{phg}}^{I} \Omega_{\mathcal{F}}^{*}(M)+\mathcal{A}^{a} \Omega_{\mathcal{F}}^{*}(M)$.

Proof. When the foliation $\mathcal{F}$ is a fibration, this is just [14, Proposition 16]. To obtain the result in the foliated case, we can apply the construction of [14] to the operator $\widetilde{D}_{\mathcal{F}}$ near $\partial \widetilde{M}$ to obtain a parametrix $\widetilde{G}_{\mathcal{F}}$. By averaging with $\Gamma$ if necessary, we can assume $\widetilde{G}_{\mathcal{F}}$ is $\Gamma$-invariant. This means (see for instance [24]) that $\widetilde{G}_{\mathcal{F}}$ descends to an operator $q_{*} \widetilde{G}_{\mathcal{F}}$ on $\partial M \times[0, \epsilon)_{x}$. This can be combined with a parametrix of $D_{\mathcal{F}}$ in the interior of $M$ to obtain a global parametrix $G_{\mathcal{F}}$ of $D_{\mathcal{F}}$, from which the result follows.

Using a similar approach, we also obtained the following generalization of [14, Proposition 17].

Proposition 23. Suppose that a is not an indicial root for $\Pi_{0} \widetilde{D}_{\mathcal{F}-c} \Pi_{0}$. Then

$$
D_{\mathcal{F}-c}: x^{a} H_{\mathcal{F}-c}^{1}(M) \rightarrow x^{a} \Pi_{0} L^{2} \Omega_{\mathcal{F}-c}^{*}(M) \oplus x^{a-1} \Pi_{\perp} L^{2} \Omega_{\mathcal{F}-c}^{*}(M)
$$

is Fredholm. If $a+1$ is not an indicial root, then

$$
D_{\mathcal{F}-c}: x^{a} \Pi_{0} H^{1} \Omega_{\mathcal{F}-c}^{*}(M) \oplus x^{a+1} \Pi_{\perp} H^{1} \Omega_{\mathcal{F}-c}^{*}(M) \rightarrow x^{a} L^{2} \Omega_{\mathcal{F}-c}^{*}(M)
$$

is Fredholm. If $D_{\mathcal{F}-c} \omega=0$, then $\omega$ is polyhomogeneous with exponents in its expansion determined by the indicial roots of $\Pi_{0} \widetilde{D}_{\mathcal{F}-c} \Pi_{0}$, while if $\eta \in \mathcal{A}^{a} \Omega_{\mathcal{F}-c}^{*}(M), \zeta \in x^{c} \Pi_{0} H^{1} \Omega_{\mathcal{F}-c}^{*} \oplus$ $x^{c+1} \Pi_{\perp} H^{1} \Omega_{\mathcal{F}-c}^{*}(M)$ for $c<a$ and $\eta=D_{\mathcal{F}-c} \zeta$, then $\zeta \in \Pi_{0} \mathcal{A}_{\mathrm{phg}}^{I} \Omega_{\mathcal{F}-c}^{*}(M)+\mathcal{A}^{a} \Omega_{\mathcal{F}-c}^{*}(M)$.

With these results, we can now relate $L^{2}$-harmonic forms with weighted $L^{2}$-cohomology. 
Theorem 24. If $\left(M, g_{\mathcal{F}}\right)$ is a manifold with a foliated boundary metric such that the boundary foliation is a good Seifert fibration (see Definition 11), then for every $k$ there is a natural isomorphism

$$
\Psi: L^{2} \mathcal{H}^{k}\left(M, g_{\mathcal{F}}\right) \rightarrow \operatorname{Im}\left(\mathcal{W} H^{k}\left(M, g_{\mathcal{F}}, \epsilon_{0}\right) \rightarrow \mathcal{W} H^{k}\left(M, g_{\mathcal{F}},-\epsilon_{0}\right)\right)
$$

for $\epsilon_{0}>0$ sufficiently small.

Proof. Thanks to Proposition 22, the proof is a straightforward adaptation of the proof of [14, Theorem 1C]. First, if $\omega \in L^{2} \mathcal{H}^{k}\left(M, g_{\mathcal{F}}\right)$, then by Proposition 22, we know $\omega$ is polyhomogeneous, so in particular is in $x^{\epsilon_{0}} L^{2} \Omega_{\mathcal{F}}^{k}(M)$ for some small $\epsilon_{0}>0$, specifically any $\epsilon_{0}$ smaller than the smallest indicial root of the Hodge-deRham operator. This gives the mapping 40. If $\Psi(\omega)=0$, then $\omega=d \zeta$ for some $\zeta \in x^{-1-\epsilon_{0}} L^{2} \Omega_{\mathcal{F}}^{k-1}(M)$. As described above, we can choose $\zeta$ to be polyhomogeneous. Then

$$
\|\omega\|^{2}=\int_{M} d \zeta \wedge * \omega=\int_{M} d(\zeta \wedge * \omega)=\lim _{x \rightarrow 0} \int_{\partial M \times\{x\}} \zeta \wedge * \omega=\frac{1}{|\Gamma|} \lim _{x \rightarrow 0} \int_{\partial M \times\{x\}} q^{*} \zeta \wedge * q^{*} \omega
$$

The latter goes to zero exactly as shown in [14].

To establish surjectivity, we note that thanks to Proposition 22 , the space of $L^{2}$-harmonic forms $L^{2} \mathcal{H}^{*}(M)$ can be identified with the cokernel of the map

$$
D_{\mathcal{F}}: x^{-\epsilon_{0}-1} \Pi_{0} H^{1} \Omega_{\mathcal{F}}^{*}(M) \oplus x^{-\epsilon_{0}} \Pi_{\perp} H^{1} \Omega_{\mathcal{F}}^{*}(M) \rightarrow x^{-\epsilon_{0}} L^{2} \Omega_{\mathcal{F}}^{*}(M)
$$

for $\epsilon_{0}>0$ small enough. This means that $x^{-\epsilon_{0}} L^{2} \Omega_{\mathcal{F}}^{*}(M)$ is equal to

$$
\operatorname{Im}\left(D_{\mathcal{F}}: x^{-\epsilon_{0}-1} \Pi_{0} H^{1} \Omega_{\mathcal{F}}^{*}(M) \oplus x^{-\epsilon_{0}} \Pi_{\perp} H^{1} \Omega_{\mathcal{F}}^{*}(M) \rightarrow x^{-\epsilon_{0}} L^{2} \Omega_{\mathcal{F}}^{*}(M)\right) \oplus L^{2} \mathcal{H}^{*}(M) .
$$

Thus, suppose $\eta \in x^{\epsilon_{0}} L^{2} \Omega_{\mathcal{F}}^{k}(M)$ is a representative for a class in the space on the right of 40. Again, we choose $\eta$ to be polyhomogeneous. According to 42, the form $\eta$ can be rewritten as

$$
\eta=D_{\mathcal{F}} \zeta+\gamma, \quad \zeta \in x^{-\epsilon_{0}-1} \Pi_{0} H^{1} \Omega_{\mathcal{F}}^{*}(M) \oplus x^{-\epsilon_{0}} \Pi_{\perp} H^{1} \Omega_{\mathcal{F}}^{*}(M), \gamma \in L^{2} \mathcal{H}^{*}(M) .
$$

Restricting this identity to the collar neighborhood $\partial M \times[0, \epsilon)_{x}$ and pulling it back to $\partial \widetilde{M} \times[0, \epsilon)_{x}$ via the quotient map $q$, we use the same argument as in [14] to conclude that

$$
\zeta=\zeta_{0}+\zeta^{\prime} \text { with } \zeta^{\prime} \in \mathcal{A}^{\epsilon_{0}} \Omega_{\mathcal{F}}^{*}(M) \text { and } \zeta_{0} \in x^{\frac{b-1}{2}}\left(\frac{\alpha_{\frac{b-1}{2}}}{x^{\frac{b-1}{2}}}+\frac{d x}{x^{2}} \wedge \frac{\beta_{\frac{b+1}{2}}}{x^{\frac{b+1}{2}}}\right)
$$

where $q^{*} \alpha_{\frac{b-1}{2}}, q^{*} \beta_{\frac{b+1}{2}} \in \operatorname{ker} \mathbb{D}$. Here, $\mathbb{D}$ is the operator defined in [14] for the metric $q^{*} g_{\mathcal{F}}$. This can be used to show, as in [14], that $\|\delta \zeta\|^{2}=0$ using integration by parts. Thus, $\delta \zeta=0$ and consequently we have that

$$
\eta=d \zeta+\gamma
$$

This shows that the cohomology class represented by $\eta$ is in the image of the map (40), establishing surjectivity.

Using Proposition 23 instead of Proposition 22 and adapting the proof of [14, Theorem 2C] in a straightforward manner, we obtain also the following. 
Theorem 25. If $\left(M, g_{\mathcal{F}-c}\right)$ is a manifold with a foliated cusp metric such that the boundary foliation is a good Seifert fibration (see Definition 11), then for every $k$ there is a natural isomorphism

$$
L^{2} \mathcal{H}_{\mathcal{F}-c}^{k}(M) \rightarrow \operatorname{Im}\left(W H^{k}\left(M, g_{\mathcal{F}-c}, \epsilon_{0}\right) \rightarrow W H^{k}\left(M, g_{\mathcal{F}-c},-\epsilon_{0}\right)\right)
$$

for $\epsilon_{0}>0$ sufficiently small.

\section{Proofs of MAIN THEOREMS}

Exactly as in [14, §5.5], Theorems 1 and 2 follow directly from Theorem 15 in light of Theorems 24 and 25. For example, for foliated cusp metrics, combining (43) and (19) gives

$$
L^{2} \mathcal{H}_{\mathcal{F}-c}^{k}(M) \simeq \operatorname{Im}\left(I H_{[\epsilon+f / 2]}^{k}(M) \rightarrow I H_{[-\epsilon+f / 2]}^{k}(M)\right)
$$

for $\epsilon>0$ sufficiently small. These are exactly the lower and upper middle perversities,

$$
\underline{\mathfrak{m}}(f+1)=\left\{\begin{array}{ll}
\frac{f-1}{2} & f \text { odd, } \\
\frac{f}{2} & f \text { even, }
\end{array} \quad \overline{\mathfrak{m}}(f+1)=\left\{\begin{array}{lll}
\frac{f-1}{2} & f & \text { odd }, \\
\frac{f}{2}-1 & f & \text { even } .
\end{array}\right.\right.
$$

The proof of Theorem 1 is similar using (18).

For Theorem 3, recall that the Witt condition is simply the assumption that for any link $L$ in a regular neighborhood, if $L$ is even dimensional, then the intersection cohomology

$$
I H_{\bar{m}}^{\operatorname{dim}} L / 2(L)=0 \text {. }
$$

In our case, a necessary and sufficient condition to insure that the space $X$ is Witt is to require that $H^{\frac{f}{2}}(\widetilde{F})=0$ (if $f$ is even). Indeed, the condition is necessary since, thanks to the fact the base $B$ is chosen to be an effective orbifold, the link of the regular neighborhoods is $\widetilde{F}$ for most of the points on $B$. To see that the condition is sufficient, notice that if $L$ is even and $H^{\frac{f}{2}}(\widetilde{F})=0$, then $(28)$ and the map $I H_{\bar{m}}^{\operatorname{dim} L / 2}(L) \hookrightarrow I H_{\bar{m}}^{\operatorname{dim}} L / 2(\widetilde{L})$ from (33) induces an injective map $I H_{\bar{m}}^{\operatorname{dim} L / 2}(L) \hookrightarrow I H_{\bar{m}}^{\operatorname{dim} L / 2}\left(C_{1}(\widetilde{F})\right)$, and $I H_{\bar{m}}^{\operatorname{dim} L / 2}\left(C_{1}(\widetilde{F})\right)$ is easily seen to be 0 by Proposition 14 and the fact $\operatorname{dim} L \geq f$. Therefore, since either $f$ is odd or $H^{\frac{f}{2}}(\widetilde{F})=0$ in the Witt case, we can just apply Theorem 15 with $a=0$ to obtain $H_{(2)}^{*}\left(M, g_{\mathcal{F}-c}\right) \cong I H_{\underline{m}}^{*}(X, B)$. By Corollary 16, we also know that the differential in the $L^{2}$-complex is closed, so that the $L^{2}$-cohomology is also naturally identified with the space of $L^{2}$-harmonic forms, completing the proof of Theorem 3. In particular, notice that this does not require the Seifert fibration $\mathcal{F}$ to be good.

\section{Some EXAMPLES}

We will now verify Theorem 1 directly for a simple example: a quotient of a multi-TaubNUT space. These are asymptotically locally flat (ALF) gravitational instantons of type $A_{k-1}$ first written down in [10]. Such a manifold, $\widetilde{M}$, contains $k$ points $p_{1}, \ldots, p_{k}$ such that $\widetilde{M}-\left\{p_{1}, \ldots, p_{k}\right\}$ is diffeomorphic to a principle $S^{1}$ bundle over $\mathbb{R}^{3}-\left\{z_{1}, \ldots, z_{k}\right\}$ of degree -1 near each $z_{i}$. The metric is locally of the form

$$
g_{A L F}^{k}=V\left(d x_{1}^{2}+d x_{2}^{2}+d x_{3}^{2}\right)+V^{-1} \eta^{2},
$$


where $\eta$ is a choice of connection 1-form for the principle $S^{1}$ bundle with curvature given by $* d V$, and

$$
V(z)=1+\sum_{i=1}^{k} \frac{1}{\left|z-z_{i}\right|} .
$$

We will assume that the $z_{i}$ lie at the points $(\cos (2 \pi / k), \sin (2 \pi / k), 0)$. The map defined by

$$
\left(x_{1}, x_{2}, x_{3}\right) \mapsto\left(\cos (2 \pi / k) x_{1}, \sin (2 \pi / k) x_{2}, x_{3}\right)
$$

generates an action of $\mathbb{Z}_{k} \subset \mathrm{SO}(3)$ on $\mathbb{R}^{3}$ which acts freely on the monopole points $z_{1}, \ldots, z_{k}$. As explained in [30, p.106], see also [16, Proposition 2.7], this action of $\mathbb{Z}_{k}$ can be lifted to an action on $\widetilde{M}$ by isometries. The lifted action is not unique, but it is once we specify the action of $\mathbb{Z}_{k}$ on the $\mathbb{S}^{1}$ fiber above the origin in $\mathbb{R}^{3}$, see again [30, p.106]. In order for $\mathbb{Z}_{k}$ to act freely on $\widetilde{M}$, we will require that the action on the $\mathbb{S}^{1}$ fibre above the origin is the one were the generator $1 \in \mathbb{Z}_{k}$ acts by multiplication by $e^{\frac{2 \pi i}{k}}$.

For this free action of $\mathbb{Z}_{k}$ by isometries on $\widetilde{M}$, we will now verify explicitly that Corollary 4 holds for

$$
M:=\widetilde{M} / \mathbb{Z}_{k}
$$

in degree 2. That is, we will show that if $X$ is the orbifold obtained as in (4) from $\bar{M}$ by collapsing the boundary foliation over the space of leaves, then

$$
L^{2} \mathcal{H}^{2}(M) \simeq H^{2}(X) .
$$

As pointed out in in [25], the 2-forms

$$
\Omega_{i}=* d V_{i}-d\left(\frac{V_{i}}{V} \eta\right), \quad V_{i}=\frac{2 m}{\left|x-p_{i}\right|}, \quad i \in\{1, \ldots, k\}
$$

give a basis of the space $L^{2} \mathcal{H}^{2}(\widetilde{M})$. The space $L^{2} \mathcal{H}^{2}(M)$ is isomorphic to the space of $\mathbb{Z}_{k^{-}}$ invariant $L^{2}$ harmonic forms on $\widetilde{M}$, which is the one-dimensional space generated by $\sum_{i=1}^{k} \Omega_{i}$. Thus $L^{2} \mathcal{H}^{2}(M) \simeq \mathbb{R}$.

Now we want to compute $H^{2}(X)$. As described in [15], the inverse image via $\widetilde{M}-$ $\left\{p_{1}, \ldots, p_{k}\right\} \longrightarrow \mathbb{R}^{3}-\left\{z_{1}, \ldots, z_{k}\right\}$ of the straight line connecting $z_{i}$ to $z_{j}$ is a nontrivial class in $H_{2}(\widetilde{M}) \simeq \mathbb{R}^{k-1}$. Let $\gamma_{i}, i=1, \ldots, k-1$, denote the Poincaré dual of the class corresponding to the line connecting $z_{i}$ to $z_{i+1}$, and $\gamma_{k}$ that for the line from $z_{k}$ to $z_{1}$. Then $\gamma_{1}, \ldots, \gamma_{k-1}$ form a basis for $H^{2}(\widetilde{M})$, and, if $k>2$ the intersections numbers are

$$
\begin{aligned}
\gamma_{i}^{2} & =-2 \\
\gamma_{i} \cdot \gamma_{j} & =1 \text { if } i-j \equiv \pm 1(\bmod k), \\
\gamma_{i} \cdot \gamma_{j} & =0 \text { otherwise. }
\end{aligned}
$$

From this, one can easily check that the intersection pairing is negative definite on $H^{2}(\widetilde{M})$ and that

$$
\sum_{i=1}^{k} \gamma_{i}=0
$$


in cohomology. If $r$ is the radial coordinate on $\mathbb{R}^{3}$, then the set $\{r>R\}$ for sufficiently large $R$ is diffeomorphic to the total space of the fibre bundle

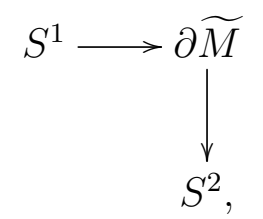

of degree $k$ crossed with $(R, \infty)_{r}$. Let $\overline{\widetilde{M}}$ be the manifold with boundary obtained from $\widetilde{M}$ by gluing the boundary $\partial \widetilde{M} \cong \mathbb{S}^{3} / \mathbb{Z}_{k}$. Let $\widetilde{X}$ the space obtained from $\overline{\widetilde{M}}$ by collapsing the fibres of the bundle 48 on the boundary of $\widetilde{\widetilde{M}}$ onto the base $S^{2}$. Since the fibres of (48) are circles, the space $\widetilde{X}$ is naturally a smooth manifold and $\widetilde{X}-\widetilde{M} \simeq S^{2}$. We claim that the de Rham cohomology of $\widetilde{X}$ satisfies $H_{d R}^{2}(\widetilde{X}) \simeq \mathbb{R}^{k}$ where a basis is given by the $\gamma_{i}, i=1, \ldots, k-1$, and the Poincaré dual of $S^{2}=\widetilde{X} \backslash \widetilde{M} \subset \widetilde{X}$, call it $\bar{\gamma}$. Indeed, we use the Mayer-Vietoris sequence for the open cover $U=\{r>R\}$ and $V=\{r<R+\epsilon\}$. The set $U \cap V$ deformation retracts onto $\partial \widetilde{M} \cong S^{3} / \mathbb{Z}_{k}$, which has trivial cohomology in degrees 1 and 2. Thus

$$
H_{d R}^{2}(\tilde{X}) \simeq H_{d R}^{2}(U) \oplus H_{d R}^{2}(V) .
$$

Since $V$ is diffeomorphic to $\widetilde{M}$ (and thus has the same homology), and since $U$ deformation retracts onto $S^{2}$, this proves our claim. The de Rham cohomology of the orbifold $X=\tilde{X} / \mathbb{Z}_{k}$ is identified by definition with the $\mathbb{Z}_{k}$-invariant part of the de Rham cohomology of $\tilde{X}$, and it is isomorphic to the singular cohomology of $X$, modulo torsion. By (47), the $\gamma_{i}$ 's have no $\mathbb{Z}_{k}$-invariant part. On the other hand, $\bar{\gamma}$ is $\mathbb{Z}_{k}$-invariant, so the space of $\mathbb{Z}_{k}$-invariant de Rham classes is one dimensional, and thus the singular cohomology of $X$ is one dimensional and (46) holds as claimed.

Of course, our results become really interesting when $M$ is not a global quotient of a manifold with fibred boundary. This is not hard to construct as the next example shows.

Example 26. Let $\mathcal{F}$ be a foliation of a closed smooth oriented 3-manifold $Y$ by circles corresponding to a good Seifert fibration on a closed surface orbifold $\Sigma$. As discussed in 24 , Example 3.5], the Seifert fibration is automatically good provided $\Sigma$ is not a tear drop or a 2-sphere with two cone points having different cone angles. We suppose of course that $\mathcal{F}$ is not a foliation coming from a fibred bundle. In this case, from Definition 11, we know that $Y=\widetilde{Y} / \Gamma$ with $\widetilde{Y}$ the total space of a circle bundle over $\widetilde{\Sigma}$ on which $\Gamma$ acts equivariantly. Moreover, the leaves of $\mathcal{F}$ are then given by the image of the fibres of the circle bundle over $\widetilde{\Sigma}$ under the quotient map.

Let $\bar{M}$ be a compact oriented manifold with boundary such that $\partial \bar{M}=Y$. Notice that since the oriented cobordism group is trivial in dimension 3 by [29], such a $\bar{M}$ always exists. We want to choose $\bar{M}$ such that the $\Gamma$-bundle $\widetilde{Y} \rightarrow Y$ does not extend to a $\Gamma$-bundle $\widetilde{M} \rightarrow M$, where $M=\bar{M} \backslash \partial \bar{M}$. In other words, if $\nu: Y \rightarrow B \Gamma$ is the corresponding map in the classifying space $B \Gamma$, we want to choose $\bar{M}$ such that $\nu$ does not extend to a map from $\bar{M}$ to BГ. To make such a ch oice, pick a loop $\gamma: \mathbb{S}^{1} \rightarrow Y$ such that $\nu \circ \gamma$ is a non-trivial element of $\pi_{1}(B \Gamma)$. Such a loop exists since we suppose the $\Gamma$-bundle $\widetilde{Y} \rightarrow Y$ is not trivial.

Clearly then, it will be impossible to extend the map $\nu$ to $\bar{M}$ if the loop $\gamma$ is contractible in $\bar{M}$. If this is not the case, move the loop $\gamma$ smoothly in the interior of $\bar{M}$ to an embedded 
loop $\gamma^{\prime}: \mathbb{S}^{1} \rightarrow M$. Since $Y$ is of dimension 3 and orientable, its tangent bundle is trivial. Consequently, the normal bundle of $\gamma\left(S^{1}\right)$ in $Y$ and of $\gamma^{\prime}\left(\mathbb{S}^{1}\right)$ in $M$ are trivial. We can thus perform a surgery of codimension 3 (see for instance [17, p.99]) along $\gamma^{\prime}\left(\mathbb{S}^{1}\right)$ in $\bar{M}$ to obtain a new oriented manifold with boundary $\bar{M}^{\prime}$ such that $\partial \bar{M}^{\prime}=Y$ and $\gamma$ is contractible in $\bar{M}^{\prime}$. In particular, the map $\nu$ does not extend to $\bar{M}^{\prime}$. Thus, $\bar{M}^{\prime}$ gives an example of a manifold with boundary foliated by good Seifert fibration for which the corresponding $\Gamma$-bundle on $\partial \bar{M}^{\prime}$ cannot be extended to $\bar{M}^{\prime}$.

\section{REFERENCES}

[1] P. Albin, E. Leichtnam, R. Mazzeo, and P. Piazza, The signature package on witt spaces, available online at arXiv:1112.0989, 2011.

[2] M. F. Atiyah, V. K. Patodi, and I. M. Singer, Spectral asymmetry and Riemannian geometry. I, Math. Proc. Cambridge Philos. Soc. 77 (1975), 43-69.

[3] A. Borel and et al., Intersection cohomology, Modern Birkhäuser Classics, Birkhäuser Boston Inc., Boston, MA, 2008, Notes on the seminar held at the University of Bern, Bern, 1983, Reprint of the 1984 edition.

[4] Jean-Paul Brasselet, Gilbert Hector, and Martin Saralegi, $\mathcal{L}^{2}$-cohomologie des espaces stratifiés, Manuscripta Math. 76 (1992), no. 1, 21-32. MR 1171153 (93i:58009)

[5] Jeff Cheeger, On the Hodge theory of Riemannian pseudomanifolds, Geometry of the Laplace operator (Proc. Sympos. Pure Math., Univ. Hawaii, Honolulu, Hawaii, 1979), Proc. Sympos. Pure Math., XXXVI, Amer. Math. Soc., Providence, R.I., 1980, pp. 91-146.

[6] Jeff Cheeger, Mark Goresky, and Robert MacPherson, L2-cohomology and intersection homology of singular algebraic varieties, Seminar on Differential Geometry, Ann. of Math. Stud., vol. 102, Princeton Univ. Press, Princeton, N.J., 1982, pp. 303-340.

[7] Weimin Chen and Yongbin Ruan, A new cohomology theory of orbifold, Comm. Math. Phys. 248 (2004), no. 1, 1-31. MR 2104605 (2005j:57036)

[8] C. Debord, J.-M. Lescure, and F. Rochon, Pseudodifferential operators on manifolds with fibred corners, available online at arXiv:1112.4575, 2011.

[9] J. J. Duistermaat and J. A. C. Kolk, Lie groups, Universitext, Springer-Verlag, Berlin, 2000.

[10] G. W. Gibbons and S. W. Hawking, Gravitational multi-instantons, Phys. Lett. B 78 (1978), no. 4, $430-432$.

[11] Sebastian Goette, Adiabatic limits of seifert fibrations, dedekind sums, and the diffeomorphism type of certain 7-manifolds, available online at arXiv:1108.5614, 2011.

[12] Mark Goresky and Robert MacPherson, Intersection homology theory, Topology 19 (1980), no. 2, 135162.

[13] Intersection homology. II, Invent. Math. 72 (1983), no. 1, 77-129.

[14] Tamás Hausel, Eugenie Hunsicker, and Rafe Mazzeo, Hodge cohomology of gravitational instantons, Duke Math. J. 122 (2004), no. 3, 485-548.

[15] N. J. Hitchin, Polygons and gravitons, Math. Proc. Cambridge Philos. Soc. 85 (1979), no. 3, $465-476$.

[16] N. Honda and J. Viaclovsky, Conformal symmetries of self-dual hyperbolic monopole metrics, available online at arXiv:1002.2119, 2010.

[17] H.B. Lawson and M-L Michelsohn, Spin geometry, Princeton Univ. Press, Princeton, 1989.

[18] Rafe Mazzeo, The Hodge cohomology of a conformally compact metric, J. Differential Geom. 28 (1988), no. 2, 309-339.

[19] Rafe Mazzeo and Ralph S. Phillips, Hodge theory on hyperbolic manifolds, Duke Math. J. 60 (1990), no. 2, 509-559.

[20] P. Molino, Riemannian foliations, Birkhäuser, Boston, 1988.

[21] Masayoshi Nagase, Sheaf theoretic $L^{2}$-cohomology, Complex analytic singularities, Adv. Stud. Pure Math., vol. 8, North-Holland, Amsterdam, 1987, pp. 273-279. MR 894298 (88g:58009)

[22] Arvind Nair, Weighted cohomology of arithmetic groups, Ann. of Math. (2) 150 (1999), no. 1, 1-31.

[23] M. Pflaum, Analytic and geometric study of stratified spaces, Lecture notes in mathematics, SpringerVerlag, Berlin, 2001. 
[24] Frédéric Rochon, Pseudodidfferential operators on manifolds with foliated boundaries, J. Funct. Anal. 262 (2012), 1309-1362.

[25] P. J. Ruback, The motion of Kaluza-Klein monopoles, Comm. Math. Phys. 107 (1986), no. 1, $93-102$.

[26] Leslie Saper and Mark Stern, L $L_{2}$-cohomology of arithmetic varieties, Ann. of Math. (2) 132 (1990), no. 1, 1-69.

[27] W. Thurston, The geometry and topology of three-manifolds, electronic edition of the 1980 notes distributed by Princeton University.

[28] Boris Vaillant, Index and spectral theory for manifolds with generalized fibred cusps, available online at arXiv: math/0102072v1, 2001.

[29] C. T. C. Wall, Determination of the cobordism ring, Ann. of Math. (2) 72 (1960), 292-311. MR 0120654 (22 \#11403)

[30] Evan P. Wright, Quotients of gravitational instantons, Ann. Global Anal. Geom. 41 (2012), no. 1, 91-108. MR 2860398

[31] Steven Zucker, $L_{2}$ cohomology of warped products and arithmetic groups, Invent. Math. 70 (1982/83), no. 2, 169-218.

Department of Mathematics, University of TOROnto

E-mail address: jgell@math.toronto.edu

DÉPARTEMENT DE MATHÉMATIQUES, UQÀM

E-mail address: rochon.frederic@uqam.ca 